\title{
Acceleration Response Estimation of a Structure on a Supportive Structure for Seismic Design"
}

\author{
Tetsuya WATANABE ${ }^{* *}$, Masatoshi IKEDA ${ }^{* * *}$ and Nobuyuki KOBAYASHI ${ }^{* * * *}$ \\ ${ }^{*}$ Saitama University \\ 255 Shimo-Okubo, Sakura-ku, Saitama 338-8570, Japan \\ E-mail:watanabe@mech.saitama-u.ac.jp \\ ***Plant Sesimic Design Systems CO., LTD. \\ 3-16-18 Fuse-Shinmachi, Kashiwa-shi, Chiba 277-0823, Japan \\ ****Aoyama Gakuin University \\ 5-10-1 Fuchinobe, Sagamihara, Kanagawa 229-8558, Japan
}

\begin{abstract}
A number of structures in industrial facilities are installed on supports, and there have been cases in which damage to such structures has been caused by coupled vibration. In seismic design procedure, the response magnification is obtained from the natural frequency and the modal damping ratio of the coupled system between the structure and the supportive structure. Therefore, the simplified estimation method for calculating the natural frequency and the modal damping ratio of the coupled system is required in the seismic design. In the past study, an equation to calculate the modal damping ratio of a coupled system was presented. The proposed equation was useful for obtaining the modal damping ratio without performing an eigenvalue analysis or a shaking test. In current seismic design procedure, for simplicity, the design response spectrum is used to estimate the acceleration response magnification of a system having one degree of freedom (1-DOF). Though the response magnification of a coupled system can be calculated based on the response magnifications of the 1 st mode and the 2 nd mode, the participation factor and modal matrix of the coupled system are required. In order to obtain the response magnification, a simpler method that does not involve the participation factor or the modal matrix is proposed, and the characteristics of the proposed method are examined.
\end{abstract}

Key words: Structure, Support, Coupled System, Estimation Modal Damping Ratio, Seismic Design

\section{Introduction}

In recent years, large-magnitude earthquakes, such as the Kobe earthquake (1995) or the Niigata-ken Chuetsu earthquake (2004), have hit structures frequently. Damage to structures in industrial facilities, such as cranes on supportive structures, is often extensive $^{(1)}$. Therefore, the seismic design of these facilities is different from the seismic design of buildings. In previous studies ${ }^{(2)-(6)}$, one of the authors proposed a response spectrum of a 1-DOF friction system. Since many of structures are erected on supportive structures, the coupled vibration of the structure and the supportive structure makes the acceleration response of the structure amplify. In the seismic design procedure, the natural

*Received 8 Jan., 2010 (No. 10-0008) [DOI: 10.1299/jsdd.4.484]

Copyright $@ 2010$ by JSME 
frequency and the modal damping ratio are calculated for the structure on a supportive structure (hereafter call as the coupled system) to obtain the response magnifications. Therefore, the natural frequency and the modal damping ratio of a coupled system must be estimated simply in order to calculate the acceleration intensity in the design stage.

Although the natural frequencies and the damping ratios of the support and the structure are estimated during the initial design or by a shaking test at the beginning of construction, it is necessary to conduct an eigenvalue analysis or a shaking test after the structure is set on the support in order to obtain the natural frequency and the modal damping ratio of the coupled system. In addition, whenever there is some modification in the structure or elements are added, an eigenvalue analysis or a shaking test must be conducted again.

The natural frequency of the coupled system is calculated theoretically in terms of the natural frequency and the damping ratio of the support and the structure, but the modal damping ratio of the coupled system is not easily obtained. Therefore, the precise estimating method for the modal damping ratio of the coupled system using the natural frequency and the damping ratio of two 1-DOF systems is desired in the seismic design procedure.

As for the coupled system with damping, eigenvalues are generally complex values, and the modal damping ratio is calculated from the ratio of the real and imaginary parts ${ }^{(7)}$. However, the theoretical expression used to calculate the modal damping ratio from the complex eigenvalue is complicated and is difficult to apply to the initial seismic design ${ }^{(8)}$.

In the past study, an equation in which the modal damping ratio of the coupled system is calculated from the damping ratios of two 1-DOF systems was proposed ${ }^{(9)}$. This equation should be useful in initial seismic design, because, the modal damping ratio is easily calculated by the presented equation without an eigenvalue analysis or a shaking test.

In order to obtain the acceleration response magnification precisely, the time history response analysis is required when the input vibration has non-stable amplitude behavior, such as in the case of an earthquake. In seismic design, the response spectrum is used to simplify and to reduce the calculation cost of the time history response analysis.

In conventional seismic design, the response spectrum analysis method is used for the coupled system. However, the modified seismic coefficient method is simpler and easier to use than the response spectrum analysis method. Since vibration frequency, mode and participation factor vary according to the mass ratio and stiffness ratio of the coupled system, the modified seismic coefficient method have not applied to the coupled system. Therefore, a simplified method to obtain easily the acceleration response magnification of the coupled system in order to estimate the seismic intensity is expected. In the present study, an estimation method is proposed and the characteristics of this method are examined comparing with several time history response analysis.

\section{Estimation of the modal damping ratio of the coupled system}

\subsection{Characteristics of the classic damping system}

The equation of motion of the coupled system with damping and without external forces, shown in Fig. 1, is given by ${ }^{(9)}$

$$
\left[\begin{array}{cc}
m_{c} & 0 \\
0 & m_{p}
\end{array}\right]\left\{\begin{array}{l}
\ddot{x}_{c} \\
\ddot{x}_{p}
\end{array}\right\}+\left[\begin{array}{cc}
c_{c} & -c_{c} \\
-c_{c} & c_{c}+c_{p}
\end{array}\right]\left\{\begin{array}{l}
\dot{x}_{c} \\
\dot{x}_{p}
\end{array}\right\}+\left[\begin{array}{cc}
k_{c} & -k_{c} \\
-k_{c} & k_{c}+k_{p}
\end{array}\right]\left\{\begin{array}{l}
x_{c} \\
x_{p}
\end{array}\right\}=\left\{\begin{array}{l}
0 \\
0
\end{array}\right\}
$$

where $m_{c}, m_{p}, c_{c}, c_{p}, k_{c}$ and $k_{p}$ are the mass of the structure, the mass of the support, the damping coefficient of the structure, the damping coefficient of the support, the spring constant of the structure, and the spring constant of the support, respectively. We define $f_{A p}\left(=\sqrt{k_{p} / m_{p}} / 2 \pi\right)$ and $f_{A c}\left(=\sqrt{k_{c} / m_{c}} / 2 \pi\right)$ as natural frequencies of the support and the structure, $\zeta_{A p}\left(=c_{p} / 2 \sqrt{m_{p} k_{p}}\right)$ and $\zeta_{A c}\left(=c_{c} / 2 \sqrt{m_{c} k_{c}}\right)$ as damping ratios of the support and the structure, and $\gamma_{\mathrm{A}}\left(=m_{c} / m_{p}\right)$ as the mass ratio. $f_{\mathrm{Ap}}, \zeta_{\mathrm{Ap}}$ and $\gamma_{\mathrm{A}}$ are different from the 
parameters in the past study ${ }^{(9)}$.

From the real eigenvalue analysis, the modal matrix is as follows:

$$
[\phi]=\left[\begin{array}{cc}
-f_{\mathrm{Ac}}^{2} & -f_{\mathrm{Ac}}^{2} \\
f_{1}^{2}-f_{\mathrm{Ac}}^{2} & f_{2}^{2}-f_{\mathrm{Ac}}^{2}
\end{array}\right]
$$

where undamped natural frequencies of the system $f_{1}$ and $f_{2}$ are given as

$$
f_{1}=\frac{\sqrt{2} f_{\mathrm{Ac}} f_{\mathrm{Ap}}}{\sqrt{\left(1+\lambda_{\mathrm{A}}\right)\left(\left(\gamma_{\mathrm{A}}+1\right) f_{\mathrm{Ac}}^{2}+f_{\mathrm{A} p}^{2}\right)}} \quad f_{2}=\frac{\sqrt{2} f_{\mathrm{Ac}} f_{\mathrm{A} p}}{\sqrt{\left(1-\lambda_{\mathrm{A}}\right)\left(\left(\gamma_{\mathrm{A}}+1\right) f_{\mathrm{Ac}}^{2}+f_{\mathrm{A} p}^{2}\right)}}
$$

and

$$
\lambda_{\mathrm{A}}=\sqrt{1-\left(\frac{2 f_{\mathrm{Ac}} f_{\mathrm{Ap}}}{\left(\gamma_{\mathrm{A}}+1\right) f_{\mathrm{Ac}}^{2}+f_{\mathrm{A} p}^{2}}\right)^{2}}
$$

When the classic damping system is assumed, we have

$$
\left[\begin{array}{cc}
c_{1} & 0 \\
0 & c_{2}
\end{array}\right]=[\phi]^{T}\left[\begin{array}{cc}
c_{c} & -c_{c} \\
-c_{c} & c_{c}+c_{p}
\end{array}\right][\phi]
$$

where $c_{1}$ and $c_{2}$ are the damping coefficients of the 1 st and 2 nd modes, respectively.

Substituting Eq.(2) into Eq.(5), the following equation is obtained:

$$
f_{1}^{2} f_{2}^{2}\left(c_{c}+c_{p}\right)-c_{p} f_{A c}^{2}\left(f_{1}^{2}+f_{2}^{2}\right)+c_{p} f_{A c}^{4}=0
$$

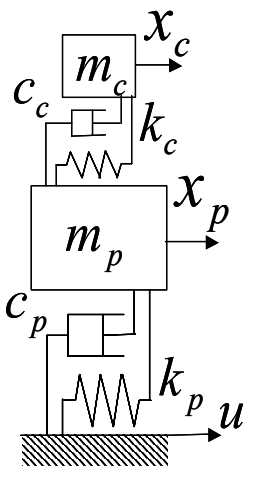

Then, from Eqs.(3) and (4), the following relations are obtained:

$$
\left.\begin{array}{c}
f_{1}^{2} f_{2}^{2}=f_{A c}^{2} f_{A p}^{2} \\
f_{1}^{2}+f_{2}^{2}=\left(\gamma_{\mathrm{A}}+1\right) f_{\mathrm{Ac}}^{2}+f_{\mathrm{Ap}}^{2}
\end{array}\right\}
$$

Substituting Eq.(7)into Eq.(6), the following equation is obtained:

$$
\frac{f_{\mathrm{A} c}}{f_{\mathrm{A} p}}=\frac{\zeta_{\mathrm{A} c}}{\zeta_{\mathrm{A} p}}
$$

Equation (8) indicates that the ratio of two natural frequencies coincides the ratio of two damping ratios in the case of a classic damping system. Here we define $R_{\mathrm{Af}}\left(=f_{\mathrm{Ac}} / f_{\mathrm{Ap}}\right)$ as the ratio of the natural frequency and $R_{A z}\left(=\zeta_{A c} / \zeta_{A p}\right)$ as the ratio of the damping ratio. Applying this relation to Eq.(3), the modal damping ratios of the 1st and 2nd modes are;

$$
\zeta_{\mathrm{A} 1 \text { (classic) }}=\frac{\sqrt{2} \zeta_{\mathrm{Ac}} \zeta_{\mathrm{Ap}}}{\sqrt{\left(1+\eta_{\mathrm{A}}\right)\left(\left(\gamma_{\mathrm{A}}+1\right) \zeta_{\mathrm{Ac}}^{2}+\zeta_{\mathrm{Ap}}^{2}\right)}} \quad \zeta_{\mathrm{A} 2 \text { (classic) }}=\frac{\sqrt{2} \zeta_{\mathrm{Ac}} \zeta_{\mathrm{Ap}}}{\sqrt{\left(1-\eta_{\mathrm{A}}\right)\left(\left(\gamma_{\mathrm{A}}+1\right) \zeta_{\mathrm{Ac}}^{2}+\zeta_{\mathrm{Ap}}^{2}\right)}}
$$

and

$$
\eta_{\mathrm{A}}=\sqrt{1-\left(\frac{2 \zeta_{A c} \zeta_{A p}}{\left(\gamma_{\mathrm{A}}+1\right) \zeta_{A c}^{2}+\zeta_{A p}^{2}}\right)^{2}}
$$

\subsection{Estimation of the modal damping ratio of a non-classic system}

The modal damping ratios of the coupled system using the damping ratios of the structure and the support is calculated from Eq.(9) in the case of a classic damping system. However, Eq.(9) cannot be applied to an actual system. Therefore, an estimation method to obtain the modal damping ratio of a non-classic damping system is proposed.

In the case of a non-classic damping system, Eq.(1) is transformed as follows ${ }^{(7)}$ : 
where

$$
\left[\begin{array}{cc}
\mathbf{C} & \mathbf{M} \\
\mathbf{M} & 0
\end{array}\right]\left\{\begin{array}{l}
\dot{x}_{c} \\
\dot{x}_{p} \\
\ddot{x}_{c} \\
\ddot{x}_{p}
\end{array}\right\}+\left[\begin{array}{cc}
\mathbf{K} & 0 \\
0 & -\mathbf{M}
\end{array}\right]\left\{\begin{array}{l}
x_{c} \\
x_{p} \\
\dot{x}_{c} \\
\dot{x}_{p}
\end{array}\right\}=0
$$

$$
\mathbf{M}=\left[\begin{array}{cc}
m_{c} & 0 \\
0 & m_{p}
\end{array}\right], \quad \mathbf{C}=\left[\begin{array}{cc}
c_{c} & -c_{c} \\
-c_{c} & c_{c}+c_{p}
\end{array}\right], \quad \mathbf{K}=\left[\begin{array}{cc}
k_{c} & -k_{c} \\
-k_{c} & k_{c}+k_{p}
\end{array}\right],
$$

and the following harmonic vibration is considered:

$$
\left\{\begin{array}{llll}
x_{c} & x_{p} & \dot{x}_{c} & \dot{x}_{p}
\end{array}\right\}^{T}=\left\{\begin{array}{llll}
X_{c} & X_{p} & \dot{X}_{c} & \dot{X}_{p}
\end{array}\right\}^{T} e^{s t} \equiv \mathbf{X}^{T} e^{s t}
$$

Equation (11) can then be rewritten as follows:

$$
\left\{\left[\begin{array}{cc}
\mathbf{C} & \mathbf{M} \\
\mathbf{M} & 0
\end{array}\right] s+\left[\begin{array}{cc}
\mathbf{K} & 0 \\
0 & -\mathbf{M}
\end{array}\right]\right\} \mathbf{X}=0
$$

The eigenvalue, $s$, for which the determinant becomes zero, is determined from the condition whereby there is no inverse matrix in the left-hand matrix, where $s$ is a complex eigenvalue. However, it is difficult to express the solution as a numerical formula because the determinant expresses a fourth-order equation. Therefore, Eq.(13) is transformed as follows:

$$
-\left[\begin{array}{cc}
\mathbf{K} & 0 \\
0 & -\mathbf{M}
\end{array}\right]^{-1}\left[\begin{array}{cc}
\mathbf{C} & \mathbf{M} \\
\mathbf{M} & 0
\end{array}\right] \mathbf{X}=\frac{1}{S} \mathbf{X}
$$

The eigenvalue of the left-hand matrix is calculated as a complex $s=a+b i$ ( $i$ : imaginary number). The modal damping ratio and the natural frequency are described as follows:

$$
\zeta=\sqrt{\frac{a^{2}}{a^{2}+b^{2}}} \quad f=-\frac{1}{2 \pi} \frac{a}{\zeta}
$$

where $a=-\zeta \omega, \quad b=\omega \sqrt{1-\zeta^{2}}$, and $\omega=2 \pi f$.

Figures 2 and 3 show the natural frequencies and the modal damping ratios of the 1 st and 2nd modes, which are calculated from Eq.(15), with respect to $R_{\mathrm{Az}}$. The straight lines in Fig. 2 indicate the systems without damping calculated from Eq.(3). The modal damping ratio of a non-classic damping system and the modal damping ratio of the classic damping system obtained by Eq.(9) are also plotted in Fig.3. These results indicate that the modal damping ratio of the non-classic damping system increases in proportion to $R_{\mathrm{Az}}$ increases and that the two curves contact at the point at which $R_{\mathrm{Az}}=R_{\mathrm{A} f}$.

Therefore, we attempt to express this line as a numerical formula. The damping ratio of the 1st mode is described by Eq.(9), as follows:

$$
\zeta_{\mathrm{A} \mid \text { (classic) }}=\frac{\sqrt{2} R_{\mathrm{A} z} \zeta_{\mathrm{A} p}}{\sqrt{\left(1+\eta_{\mathrm{A}}\right)\left(1+\left(1+\gamma_{\mathrm{A}}\right) R_{\mathrm{Az}}^{2}\right)}}
$$

By differentiating Eq. (16) with respect to $R_{\mathrm{A} z}$, the incline at $R_{\mathrm{Az}}=R_{\mathrm{A} f}$ is obtained.

$$
\left(\frac{d \zeta_{\mathrm{A} \mid \text { (classic) }}}{d R_{\mathrm{A} z}}\right)_{R_{A z}=R_{A}}=\frac{\eta_{\mathrm{A}}+1+\left(\eta_{\mathrm{A}}-1\right)\left(1+\gamma_{\mathrm{A}}\right) R_{\mathrm{Af}}^{2}}{\sqrt{2} \eta_{\mathrm{A}} \sqrt{\left(1+\eta_{\mathrm{A}}\right)\left(1+\left(1+\gamma_{\mathrm{A}}\right) R_{\mathrm{A} f}^{2}\right)^{3}}} \zeta_{\mathrm{A} p}
$$

The tangential line is calculated as follows:

$$
\zeta_{\mathrm{Al}}=\left(\frac{d \zeta_{\mathrm{Al} \text { (classic) }}}{d R_{\mathrm{A} z}}\right)_{R_{N_{\mathrm{A}}=R_{\mathrm{A}}}} R_{\mathrm{Az}}+c
$$


Then, the intercept $c$ is obtained as follows:

$$
c=\frac{\eta_{\mathrm{A}}-1+\left(\eta_{\mathrm{A}}+1\right)\left(1+\gamma_{\mathrm{A}}\right) R_{\mathrm{A} f}^{2}}{\sqrt{2} \eta_{\mathrm{A}} \sqrt{\left(1+\eta_{\mathrm{A}}\right)\left(1+\left(1+\gamma_{\mathrm{A}}\right) R_{\mathrm{A} f}^{2}\right)^{3}}} R_{\mathrm{A} f} \zeta_{\mathrm{A} p}
$$

The modal damping ratio of the non-classic damping system can be calculated easily by these equations without an eigenvalue analysis or a shaking test.

$$
\begin{aligned}
& \zeta_{\mathrm{A} 1}=\frac{\zeta_{\mathrm{A} p}}{\sqrt{2} \eta_{\mathrm{A}} \sqrt{\left(1+\eta_{\mathrm{A}}\right)\left(1+\left(1+\gamma_{\mathrm{A}}\right) R_{\mathrm{A} f}^{2}\right)^{3}}}\left[\left\{\eta_{\mathrm{A}}+1+\left(\eta_{\mathrm{A}}-1\right)\left(1+\gamma_{\mathrm{A}}\right) R_{\mathrm{A} f}^{2}\right\} R_{\mathrm{A} z}+\left\{\eta_{\mathrm{A}}-1+\left(\eta_{\mathrm{A}}+1\right)\left(1+\gamma_{\mathrm{A}}\right) R_{\mathrm{A} f}^{2}\right\} R_{\mathrm{A} f}\right] \\
& \zeta_{\mathrm{A} 2}=\frac{\zeta_{\mathrm{A} p}}{\sqrt{2} \eta_{\mathrm{A}} \sqrt{\left(1-\eta_{\mathrm{A}}\right)\left(1+\left(1+\gamma_{\mathrm{A}}\right) R_{\mathrm{A} f}^{2}\right)^{3}}}\left[\left\{\eta_{\mathrm{A}}-1+\left(\eta_{\mathrm{A}}+1\right)\left(1+\gamma_{\mathrm{A}}\right) R_{\mathrm{A} f}^{2}\right\} R_{\mathrm{A} z}+\left\{\eta_{\mathrm{A}}+1+\left(\eta_{\mathrm{A}}-1\right)\left(1+\gamma_{\mathrm{A}}\right) R_{\mathrm{A} f}^{2}\right\} R_{\mathrm{A} f}\right]
\end{aligned}
$$

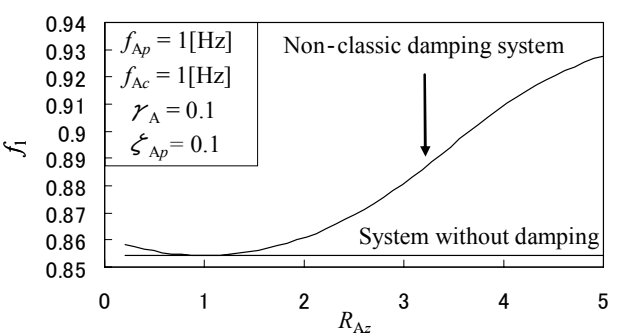

(a) First mode

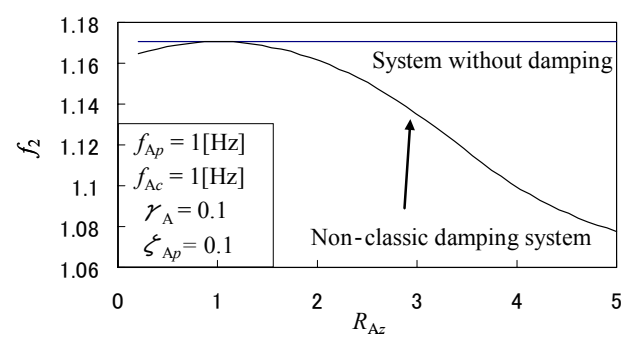

(b) Second mode

Fig. 2 Modal frequency with respect to $R_{\mathrm{Az}}$

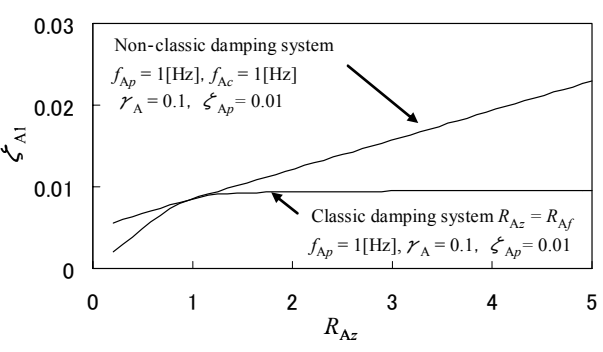

(a) First mode

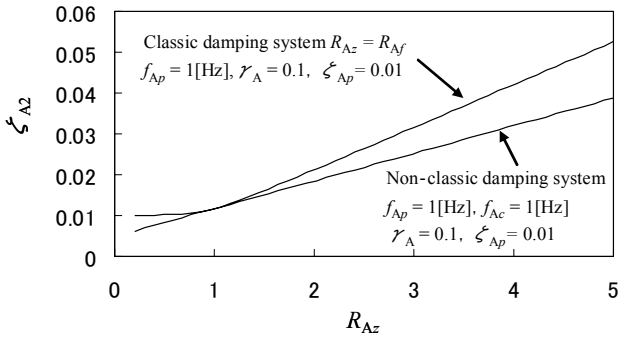

(b) Second mode

Fig. 3 Modal damping ratio with respect to $R_{\mathrm{A} z}$

Figure 4 illustrates a design spectrum as the acceleration response magnification of the 1 -DOF system when $\zeta$ is 0.05 in the seismic design guideline of a high-pressure gas facility ${ }^{(8)}$. Four acceleration response magnification polygonal lines are provided in the guideline according to the ground characteristics. The abscissa axis is the natural period of the system, and the vertical axis is the acceleration response magnification $R_{A}$. When the damping ratio calculated by using Eq.(20) and Eq.(21) is different from $0.05, R_{A}$ is multiplied by the coefficient in table 1 to correct the damping effect on the response magnification.

$$
R_{A}=\frac{\text { Maximum acceleration response }}{\text { Maximum acceleration of input wave }}
$$




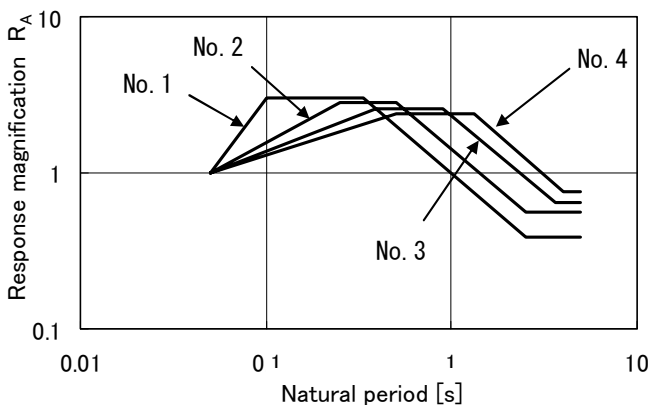

Fig. 4 Design response spectrum

Table 1 Revision coefficient

\begin{tabular}{|c|c|c|c|c|c|c|c|}
\hline $\begin{array}{c}\text { Damping } \\
\text { ratio }\end{array}$ & 0.01 & 0.02 & 0.03 & 0.05 & 0.10 & 0.15 & 0.20 \\
\hline Coefficient & 1.55 & 1.32 & 1.18 & 1.00 & 0.78 & 0.65 & 0.56 \\
\hline
\end{tabular}

\section{Response magnification of the coupled system in seismic design}

\subsection{Acceleration response magnification}

The equation of motion of the coupled system under the base excitation $u$ is derived from Eq.(1) as:

$$
\left.\begin{array}{l}
m_{c} \ddot{x}_{c}+c_{c}\left(\dot{x}_{c}-\dot{x}_{p}\right)+k_{c}\left(x_{c}-x_{p}\right)=0 \\
m_{p} \ddot{x}_{p}-c_{c}\left(\dot{x}_{c}-\dot{x}_{p}\right)-k_{c}\left(x_{c}-x_{p}\right)+c_{p}\left(\dot{x}_{p}-\dot{u}\right)+k_{p}\left(x_{p}-u\right)=0
\end{array}\right\}
$$

In the case that the coupled system is under the base excitation $u$, Eq.(24) is modified using relative displacements, $z_{c}=x_{c}-u, z_{p}=x_{p}-u$, and parameters $T_{c}=1 / f_{A c}=2 \pi \sqrt{m_{c} / k_{c}}$, $T_{p}=1 / f_{p}=2 \pi \sqrt{\left(m_{c}+m_{p}\right) / k_{p}}, \gamma=m_{c} /\left(m_{c}+m_{p}\right), \zeta_{c}=\zeta_{A c}=c_{c} / 2 \sqrt{m_{c} k_{c}}, \quad \zeta_{p}=c_{p} / 2 \sqrt{\left(m_{c}+m_{p}\right) k_{p}}$, $R_{f}=f_{c} / f_{p}, R_{z}=\zeta_{c} / \zeta_{p}$,

$$
\left.\begin{array}{l}
\ddot{z}_{c}+4 \pi \frac{\zeta_{c}}{T_{c}}\left(\dot{z}_{c}-\dot{z}_{p}\right)+\left(\frac{2 \pi}{T_{c}}\right)^{2}\left(z_{c}-z_{p}\right)=-\ddot{u} \\
\ddot{z}_{p}-4 \pi \frac{\zeta_{c}}{T_{c}} \frac{\gamma}{1-\gamma}\left(\dot{z}_{c}-\dot{z}_{p}\right)-\left(\frac{2 \pi}{T_{c}}\right)^{2} \frac{\gamma}{1-\gamma}\left(z_{c}-z_{p}\right)+4 \pi \frac{\zeta_{p}}{T_{p}} \frac{1}{1-\gamma} \dot{z}_{p}+\left(\frac{2 \pi}{T_{p}}\right)^{2} \frac{1}{1-\gamma} z_{p}=-\ddot{u}
\end{array}\right\}
$$

The equivalent modal damping ratios shown in Section 2 are modified as follows ${ }^{(9)}$.

$$
\begin{aligned}
& \zeta_{1}=\frac{\zeta_{p}}{\sqrt{2} \eta \sqrt{(1+\eta)\left(1+R_{f}^{2}\right)^{3}}}\left[\left\{(1+\eta)\left(1+R_{f}^{2}\right)-2 R_{f}^{2}\right\} R_{z}+R_{f}\left(1+R_{f}^{2}\right)(\eta-1)+2 R_{f}^{3}\right] \\
& \zeta_{2}=\frac{\zeta_{p}}{\sqrt{2} \eta \sqrt{(1-\eta)\left(1+R_{f}^{2}\right)^{3}}}\left[\left\{-(1-\eta)\left(1+R_{f}^{2}\right)+2 R_{f}^{2}\right\} R_{z}+R_{f}\left(1+R_{f}^{2}\right)(\eta+1)-2 R_{f}^{3}\right] \\
& \eta=\sqrt{1-(1-\gamma)\left(\frac{2 R_{f}}{R_{f}^{2}+1}\right)^{2}}
\end{aligned}
$$

First and second undamped natural periods $T_{1}$ and $T_{2}$, and their natural modes are:

$$
\left.\begin{array}{l}
T_{1}=\sqrt{(1+\lambda) \frac{T_{c}^{2}+T_{p}^{2}}{2}} \\
T_{2}=\sqrt{(1-\lambda) \frac{T_{c}^{2}+T_{p}^{2}}{2}}
\end{array}\right\} \quad \lambda=\sqrt{1-(1-\gamma)\left(\frac{2 T_{c} T_{p}}{T_{c}^{2}+T_{p}^{2}}\right)^{2}}
$$




$$
\frac{x_{c 1}}{x_{p 1}}=\frac{1}{1-\left(\frac{T_{c}}{T_{1}}\right)^{2}}, \quad \frac{x_{c 2}}{x_{p 2}}=\frac{1}{1-\left(\frac{T_{c}}{T_{2}}\right)^{2}}
$$

where $x_{c 1}$ and $x_{p 1}$ are the 1st modes of the structure and the support, and $x_{c 2}$, and $x_{p 2}$ are 2nd modes of them, respectively. The participation factors $I_{1}$ and $I_{2}$ for each mode are obtained as follows:

$$
I_{1}=\frac{m_{c} x_{c 1}+m_{p} x_{p 1}}{m_{c} x_{c 1}^{2}+m_{p} x_{p 1}^{2}}=\frac{1}{1-\left(\frac{T_{1}}{T_{2}}\right)^{2}} \cdot \frac{1}{x_{c 1}}, \quad I_{2}=\frac{m_{c} x_{c 2}+m_{p} x_{p 2}}{m_{c} x_{c 2}^{2}+m_{p} x_{p 2}^{2}}=\frac{1}{1-\left(\frac{T_{2}}{T_{1}}\right)^{2}} \cdot \frac{1}{x_{c 2}}
$$

The response acceleration of the structure on the support $a_{c}$ is obtained by the SRSS method using the maximum acceleration of the ground $\alpha$, and the design response magnifications of the 1 st and 2 nd modes $\beta_{1}$ and $\beta_{2}$.

$$
a_{c}=\sqrt{\left(I_{1} x_{c 1} \beta_{1}\right)^{2}+\left(I_{2} x_{c 2} \beta_{2}\right)^{2}} \cdot \alpha
$$

Since the difference between $\beta_{1}$ and $\beta_{2}$ gives small effect on the response acceleration. We put $\beta=\max \left(\beta_{1}, \beta_{2}\right)$. Equation (31) is then approximated as follows:

$$
a_{c}=\sqrt{\left(I_{1} x_{c 1}\right)^{2}+\left(I_{2} x_{c 2}\right)^{2}} \cdot \beta \alpha
$$

The response acceleration of the structure on the support $a_{c}$ is estimated from Eq.(33) when the design response spectrum is given.

\subsection{Sin 4 wave response}

We consider the response magnification $R_{A s}$ calculated from Eq.(23), by the sinusoidal input of four waves ${ }^{(8)}$, whose period coincides with the natural frequency of the 1-DOF system to determine the corrective factor on the response magnification of the coupled system. Hereafter we call $R_{A s}$ as "Sin 4 wave response". Figure 5 compares $R_{A s}$ and $R_{A}$ using six past recorded earthquakes with $\zeta=0.05$. The earthquakes shown in this figure are the Niigata earthquake NS (1964), the Tokachi earthquake NS (1968), the Hyogoken-Nanbu earthquake JR Takatori NS (1995), the Imperial Valley earthquake El Centro NS (1940), Niigata-ken Cyuetsu earthquake Ojiya NS (2004) and the Kern County earthquake N21E (1952). It is found that $R_{A s}$ is much larger than $R_{A}$ calculated by any of the recorded earthquakes and constant value. Moreover the large response magnification yields large seismic inertia force, we use the $\sin 4$ wave response to derive the response magnification of the coupled system.

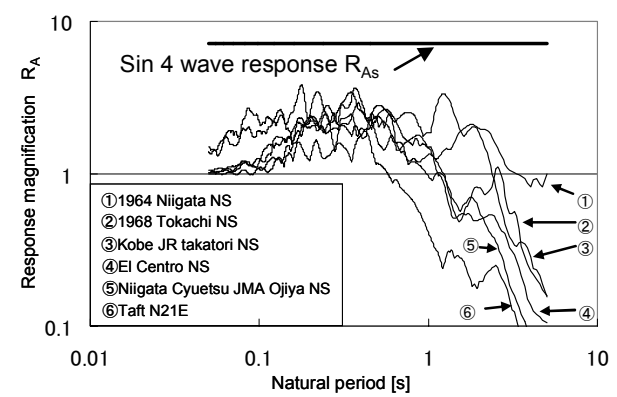

Fig. 5 Response acceleration spectrum 


\subsection{Method for estimating the response magnification of the coupled system}

When the mass ratio $\gamma$ is very small, we put $\gamma=0.0$ in Eq.(25), the system comes to be decoupled, and Eq.(25) is rewritten using $z_{c p}=z_{c}-z_{p}$.

$$
\left.\begin{array}{c}
\ddot{z}_{c p}+4 \pi \frac{\zeta_{c}}{T_{c}} \dot{z}_{c p}+\left(\frac{2 \pi}{T_{c}}\right)^{2} z_{c p}=-\ddot{x}_{p} \\
\ddot{z}_{p}+4 \pi \frac{\zeta_{p}}{T_{p}} \dot{z}_{p}+\left(\frac{2 \pi}{T_{p}}\right)^{2} z_{p}=-\ddot{u}
\end{array}\right\}
$$

Figure 6 displays the relation between $\zeta_{c}$ and $R_{A s}$ when we put $\gamma=0.0, T_{p}=T_{c}=1 \mathrm{sec}$ and $\zeta_{p}=0.05$. It is found that $R_{A s}$ is large value because the structure is excited by the resonant response of the support. Therefore, the response magnification of the structure becomes larger when the natural periods of the support close to that of the structure. As such, the corrective factor is required in $a_{c}$ when the mass ratio is small.

Substituting Eq.(31) into Eq. (33), we obtain the following:

$$
a_{c}=\sqrt{\frac{1+\left(\frac{T_{2}}{T_{1}}\right)^{4}}{\left\{1-\left(\frac{T_{2}}{T_{1}}\right)^{2}\right\}^{2}}} \cdot \beta \alpha
$$

We put Eq.(35) as Eq.(36) and hypothesize the corrective factor $\delta$ as Eq.(37) referring the damping steady state response of the 1-DOF system.

$$
a_{c}=\delta \beta \alpha
$$

$$
\delta=\sqrt{\frac{1+\left(\frac{T_{2}}{T_{1}}\right)^{4}}{\left\{1-\left(\frac{T_{2}}{T_{1}}\right)^{2}\right\}^{2}+4 \kappa^{2}\left(\frac{T_{2}}{T_{1}}\right)^{2}}}
$$

Here, $\kappa$ gives the equivalent damping of the coupled system and is obtained by the response magnification of the 1-DOF system for the case of the $\sin 4$ wave response. The relationship between the damping ratio $\zeta_{c}$ of the structure and $\kappa$ is shown in Fig. 7.

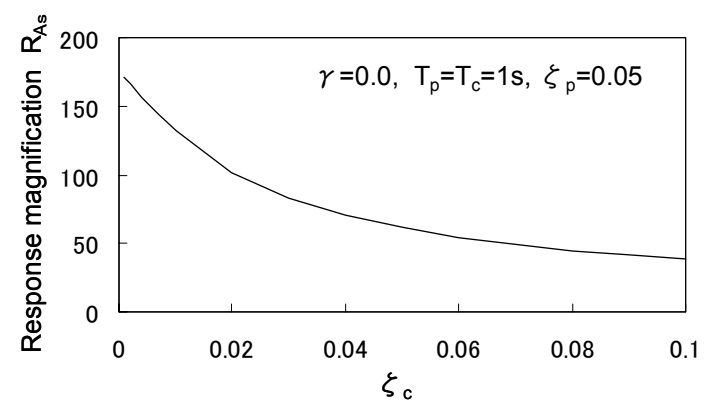

Fig. 6 Response of the 2-DOF system

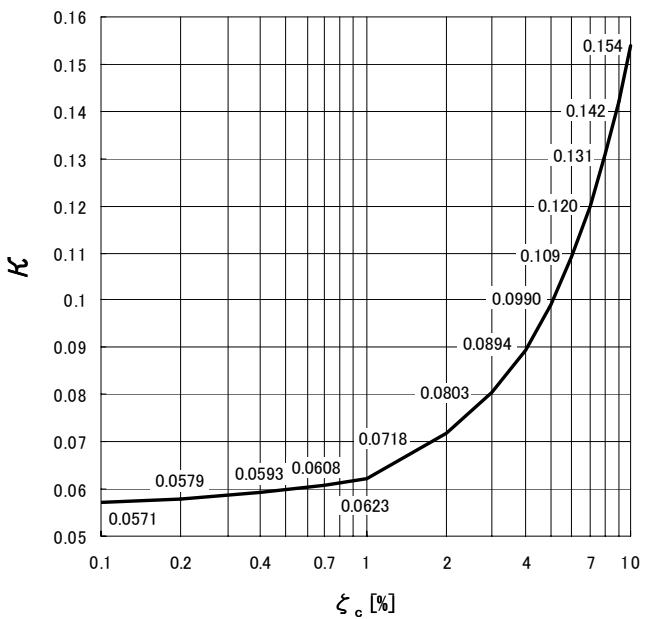

Fig. 7 Plot of $\kappa$ with respect to $\zeta_{\mathrm{c}}$

Substituting Eq.(29) into Eq.(37), the corrective factor $\delta$ which corrects the acceleration 
response magnification of the structure on the support with respect to the ground can be obtained as Eq.(38).

$$
\delta=\sqrt{\frac{1}{2} \cdot \frac{1+\lambda^{2}}{\lambda^{2}+\left(1-\lambda^{2}\right) \kappa^{2}}} \cong 0.71 \sqrt{\frac{1+\lambda^{2}}{\lambda^{2}+\left(1-\lambda^{2}\right) \kappa^{2}}}
$$

To account for the effect on the structural frequency variation of the possible uncertainties in the material properties of the structure, support and soil, we broaden the frequency of the spectrum peak $\pm 10 \%$ around $T_{c}=T_{p}$. Then $\lambda$ in Eq.(29) is modified as Table 2. Figures 8 and 9 display $\lambda$ and $\delta$ with respect to the frequency ratio $T_{c} / T_{p}$ and the mass ratio $\gamma$. It is found that the corrective factor increases when $T_{c} / T_{p}$ closes to 1 and the mass ratio is small.

Table 2 Modification of $\lambda$

\begin{tabular}{|c|c|}
\hline The natural frequency ratio & $\lambda$ \\
\hline$T_{c} / T_{p} \leqq 0.9$ & $\sqrt{1-(1-\gamma)\left(\frac{1.8 T_{c} T_{p}}{T_{c}^{2}+0.81 T_{p}^{2}}\right)^{2}}$ \\
\hline $0.9<T_{c} / T_{p} \leqq 1.1$ & $\sqrt{\gamma}$ \\
\hline$T_{c} / T_{p}>1.1$ & $\sqrt{1-(1-\gamma)\left(\frac{2.2 T_{c} T_{p}}{T_{c}^{2}+1.21 T_{p}^{2}}\right)^{2}}$ \\
\hline
\end{tabular}

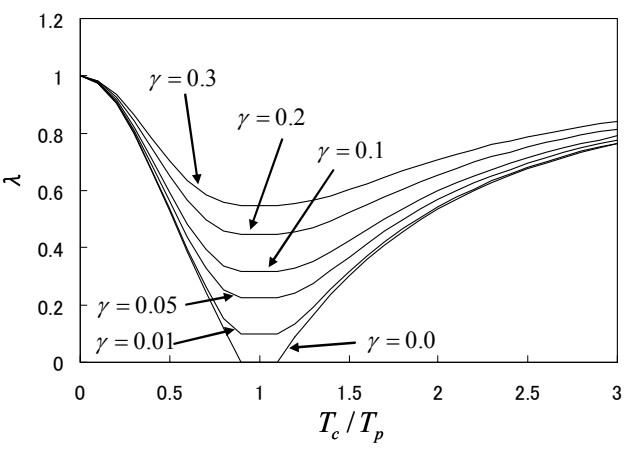

Fig.8 $\lambda$ with respect to $T_{c} / T_{p}$

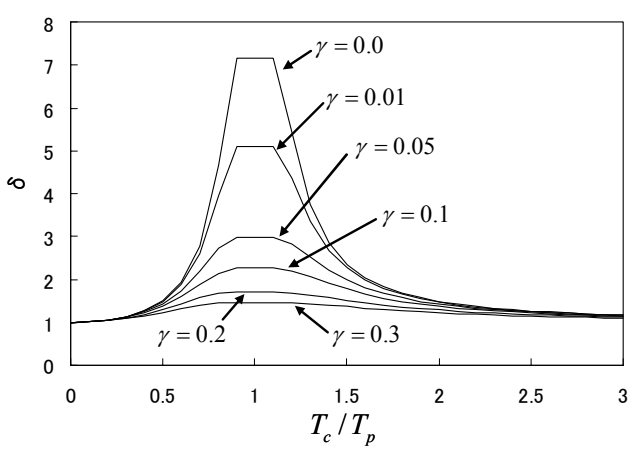

Fig.9 $\delta$ with respect to $T_{c} / T_{p}$

\subsection{Examination of the proposed method}

To examine the validity of the proposed method which is expressed by Eq.(38) and Table 2, we carried out the time history response simulation with three recorded earthquake accelerations, the El Centro NS wave, the JR Takatori NS wave, and the Niigata NS wave. Figure 10 compares $\delta \beta$ and $R_{A}$ with respect to $\zeta_{c}$ in the case $T_{p}=T_{c}=1 \mathrm{sec}, \kappa=0.099$, and $\beta=3$. The thick line plots $\delta \beta$ using Eq.(38), and the thin lines plot $R_{A}$ by the time history analysis. When the mass ratio $\gamma$ is zero, $\delta \beta$ is much larger than $R_{A}$. On the other hand, when the mass ratio is $0.1, \delta \beta$ closes to $R_{A}$ of the JR Takatori NS wave that is the largest among three waves, and $\delta \beta$ becomes much smaller than $\delta \beta$ of $\gamma=0$.

Figure 11 illustrates the effect of the combination between $T_{c}$ and $T_{p}$ on $\delta \beta$ and $R_{A}$ in the case of $\zeta_{p}=\zeta_{c}=0.05, \kappa=0.099, \gamma=0.01$ and $\beta=3 . R_{A}$ obtained by the time history analysis for the JR Takatori NS wave is shown in Fig. 11(a), and $\delta \beta$ obtained by Eq.(38) is also shown in Fig. 11(b). It is found that $\delta \beta$ and $R_{A}$ become large when $T_{c}$ closes to $T_{p}$, that 
is near the resonance. We define the ratio of the response magnification $R_{m a g}$ as "The Response magnification ratio" and described as Eq. (39).

$$
R_{\text {mag }}=\frac{\text { The response magnification of the time history analysis, } R_{\mathrm{A}}}{\text { The response magnification of the proposed estimation method, } \delta \beta}
$$

Figure 11(c) plots $R_{\text {mag }}$, and it is found that $R_{\text {mag }}$ is less than 1 . This result indicates that the response magnification used by $\delta \beta$ gives the safety side value than by $R_{A}$.

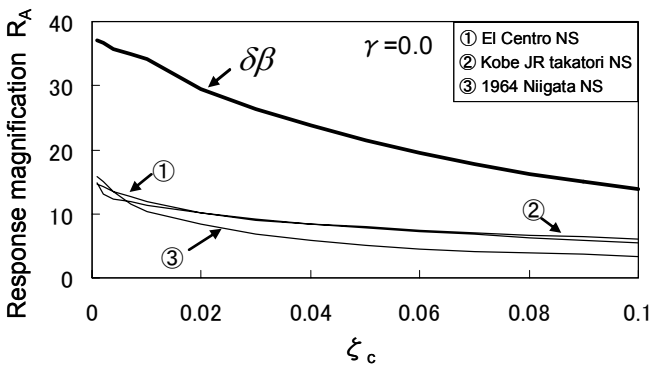

(a) $\gamma=0$

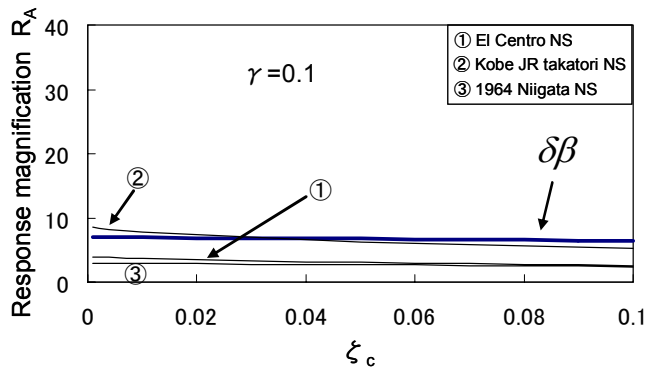

(b) $\gamma=0.1$

Fig. 10 Response magnification of the coupled system

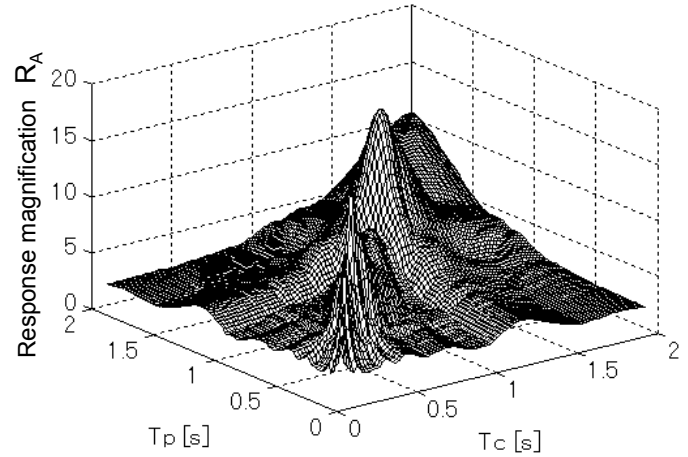

(a) Response magnification obtained by the time history analysis

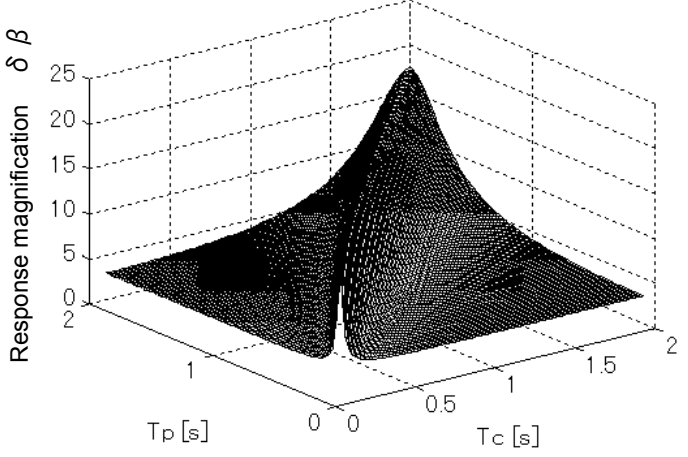

(b) Response magnification obtained by the proposed estimation method

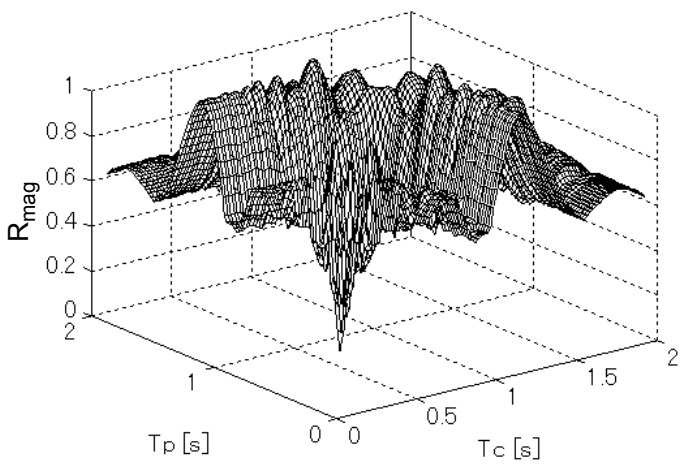

(c) Response magnification ratio

Fig. 11 The response magnification with respect to $T_{p}$ and $T_{c}$ 


\section{Conclusions}

We presented two simplified methods relating to the seismic design of the coupled system that is composed of the structure and its supportive structure. One is an estimating method for calculating the modal damping ratio from the damping ratios of the support and the structure without an eigenvalue analysis or a shaking test. The other is a simplified method for estimating the acceleration response magnification of the structure on the support with respect to the ground acceleration without an eigenvalue analysis. The proposed method is verified to estimate well the acceleration response magnification of the structure on the support, comparing to the acceleration response magnification of the structure obtained by the time history earthquake response analysis.

\section{References}

(1) Editorial Committee for the Report on the Hanshin-Awaji Earthquake Disaster, Report on the Hanshin-Awaji Earthquake Disaster, Maruzen, (1998).

(2) Watanabe, T., Mitsumori, T., Suzuki, K., Shimizu, N., Ogawa, N., Minowa, C., Vibration Test of Plant Piping System Having Friction Support, Transactions of the Japan Society of Mechanical Engineers, Series C, Vol. 58, No. 552 (1992), pp. 17-23.

(3) Watanabe, T., Suzuki, K., Mitsumori, T., Shimizu, N., Seismic Responses of Plant Piping System Having Friction and Vibro-impact Characteristics, Transactions of the Japan Society of Mechanical Engineers, Series C, Vol. 62, No. 595 (1996), pp. 63-68.

(4) Watanabe, T., Suzuki, K., Seismic Responses of Piping System with Friction Sliding on a Plane, Transactions of the Japan Society of Mechanical Engineers, Series C, Vol. 64, No. 617 (1998), pp. 64-69.

(5) Watanabe, T., Suzuki, K., Seismic Response Analysis of Piping System with Friction Support (Maximum Response Estimation Using Frictional Response Spectrum), Transactions of the Japan Society of Mechanical Engineers, Series C, Vol. 64, No. 619 (1998), pp. 87-92.

(6) Watanabe, T., Suzuki, K., Estimation of Equivalent Damping Ratio using Friction Response Spectrum, Transactions of the Japan Society of Mechanical Engineers, Series C, Vol. 66, No. 642 (2000), pp. 87-92.

(7) Nagamatsu, A., Modal Analysis, Baifukan, pp. 76-79.

(8) KHK Seismic Design of High Pressure Gas Facility, (1983).

(9) Watanabe, T., Tomoda, A., Tanaka, K., Estimation of the Damping Ratio of the Coupled System Based on the Damping Ratios of Two 1-dof Systems, JSME Journal of System Design and Dynamics, Vol.2,No.4,(2008). 